\title{
BASES IN VECTOR SPACES AND THE AXIOM OF CHOICE ${ }^{1}$
}

\author{
JAMES D. HALPERN
}

1. Because of the nonconstructive nature of the axiom of choice there has been much interest in how much of it is needed for various theories. In the case of the theory of vector spaces it appears that one would want to save at least the following two consequences of AC: (1) Every vector space has a basis and (2) Any two bases of a given vector space are equipollent. The question immediately arises: Have we saved the whole axiom of choice; namely is the axiom of choice a logical consequence of (1) and (2) and the other axioms of some appropriate set theory? This question remains open and the author conjectures a negative solution. However, we are able to show that a reasonable strengthening of (1), which is also a consequence of $\mathrm{AC}$, implies $\mathrm{AC}$, namely the universal generalization of Proposition 2 of [1], which we will call the downward basis principle:

(DB) Every vector space $V$ has the property that every subset of $V$ which generates $V$, includes a basis. ${ }^{2}$

That AC implies (DB) is a consequence of Zorn's lemma; a maximal linearly independent subset of a generating set is a basis of $V$.

We take $\mathrm{AC}$ in the following form: If $Y$ is a disjoint set of nonempty sets there is a set $S$ which has exactly one element in common with each set in $Y$.

Proof that (DB) implies AC. Notation: $U Y$ denotes $\{x: \exists X \in$ $Y[x \in X]\},|X|$ denotes the cardinality of $X, 0$ ambiguously denotes the additive field identity and the zero vector.

Let $Y$ be a disjoint set of nonempty sets. First we construct a field $F$ such that $|\cup Y| \leqq|F-\{0\}|$ : Let $R$ be any field and let $F$ be the field obtained from $R$ by adjoining $U Y$ to $R$ as a set of transcendentals. By this we actually mean the quotient field of the ring whose construction is that given by Zariski-Samuel [8, p. 40], second and

1 Presented to the Society, April 12, 1965; received by the editors September 24, 1965.

2 My thanks to M. N. Bleicher for posing the problem which we here solve positively. In [1] Bleicher credits the author with proving $\mathrm{AC}$ from the statement that for any fixed $p$ ( $p$, a prime or 0$)$, every vector space $V$ over a field of characteristic $p$ has the property that every subset of $V$ which generates $V$.includes a basis. The proof given here does actually accomplish this, and in fact, as the referee has remarked, "field" can be replaced by "pure transcendental field." 
third paragraphs, with their $E$ construed as $U Y$. This construction is easily carried out in any of the familiar formalizations of set theory and in no way involves the axiom choice. Let

$$
\text { *: UY } \underset{\text { into }}{\stackrel{1-1}{\longrightarrow}} F-\{0\} \text {. }
$$

Thus $x, y \in \cup Y \Rightarrow x^{*}, y^{*} \in F$ and $x^{*} \neq 0$. Also, if $x \neq y$ then $x^{*} \neq y^{*}$.

Let $F^{\left[U_{Y]}\right.}$ be the set of functions whose domains are $U Y$ and whose ranges are included in $F$ and such that $\{x \in U Y: f(x) \neq 0\}$ is finite. Let $V$ be the vector space of $F^{\left[U_{Y}\right]}$ over $F$, i.e. vector addition is defined by

$$
(f+g)(x)=f(x)+g(x) \text { all } f, g \in F^{[U Y]}, x \in U Y,
$$

and scalar multiplication is defined by

$$
(\alpha f)(x)=\alpha f(x) \quad \text { all } \alpha \in F, f \in F^{[U Y]}, x \in U Y .
$$

If $\alpha \in F$ and $x \in U Y$, let $(x / \alpha)$ be the function $f$ in $F^{\left[U_{Y]}\right.}$ such that $f(x)=\alpha, f(y)=0$ all $y \neq x$.

Note. $\{(x / 1): x \in \bigcup Y\}$ is a basis for $V$. For any $v \in V, x \in U Y$ we let $v_{x}$ be the $(x / 1)$ component of $v$ relative to this basis. Thus

$$
\sum_{x \in \mathbf{U} Y ; v_{x} \neq 0} v_{x} \cdot(x / 1)=v .
$$

Let $W$ be the subspace of $V$ generated by

$$
\left\{x^{*}(y / 1)-y^{*}(x / 1): \exists X \in Y[x, y \in X \text { and } x \neq y]\right\} .
$$

Lemma. If $v \in W$ then

$$
\sum_{x \in X ; v_{x \neq 0}} x^{*} v_{x}=0 \text { all } X \in Y
$$

Proof. Any element of $W$ is of the form

$$
\sum_{i=1}^{n} \alpha_{i}\left(x_{i}^{*}\left(y_{i} / 1\right)-y_{i}^{*}\left(x_{i} / 1\right)\right) .
$$

The lemma is easily proven by induction on $n$.

As a corollary we conclude: (1) If $x \in X \in Y$ and $v_{z}=0$ all $z \in X$ then $(x / 1)-v \notin W$, (since $\left.x^{*} \neq 0\right)$.

Let $V_{1}=V / W$ and let $\bar{x}$ denote $(x / 1)+W$, any $x \in U Y$. Since $V$ is generated by $\{(x / 1): x \in \cup Y\}, V_{1}$, is generated by $\{\bar{x}: x \in \cup Y\}$. 
Applying (DB) we obtain a basis $S \subseteq\{\bar{x}: x \in \cup Y\}$. Let $C=\{x \in \cup Y$ : $\bar{x} \in S\}$. We claim $C$ is a choice set for $Y$ : Let $X \in Y$ and suppose $x, y \in C \cap X$. Then $\bar{x}, \bar{y} \in S$. If $x \neq y$, then $y^{*} \bar{x}=x^{*} \bar{y}$ and hence $\bar{x}, \bar{y}$ are distinct (since $x^{*} \neq y^{*}$ ) and dependent, contrary to $S$ being a basis. Thus $x=y$, i.e. $C \cap X$ has at most one element. $C \cap X$ has at least one element since $S$ generates $V_{1}$ and (1). Thus $C$ is indeed a choice set for $Y$. Q.E.D.

Added in proof. Hans Läuchli has suggested a simplification of this proof as follows: Let $V$ be the vector space $F^{[Y]}$ (instead of $F^{[\cup Y]}$ ) over $F$. For $x \in \cup Y$ let

$$
\bar{x}(X)=\left\{\begin{array}{l}
x^{*} \text { if } x \in X \\
0 \text { otherwise }
\end{array}\right\} \quad \text { all } X \in Y .
$$

Then $\{\bar{x}: x \in \cup Y\}$ generates $V$. If $S$ is a basis included in this set then $C=\{x \in \cup Y: \bar{x} \in S\}$ is seen to be the required choice set.

2. In the other direction, it is not difficult, in the light of known results, to show that (2) by itself does not imply the axiom of choice in the context of full (i.e. with the axiom of regularity) ZermeloFraenkel set theory. It suffices to show that (2) is a consequence of the Boolean prime ideal theorem and then appeal to the, as yet unpublished, result of Halpern, Läuchli, and Lévy that the Boolean prime ideal theorem does not imply AC in said context. ${ }^{3}$

Marshall Hall [2] has shown that (2) is a consequence of Philip Hall's theorem on sets of distinct representatives extended to infinite families which reads as follows: If $Y$ is a family of finite sets such that any $k$ sets in $Y$ have at least $k$ elements in their union ( $k$ any finite number) then there is a 1-1 choice function for $Y$, i.e. a 1-1 function with domain $Y$ such that $f(X) \in X$ all $X \in Y$. This in turn is a consequence of Philip Hall's theorem for finite families $Y$ and the Tychonoff theorem for $T_{2}$-spaces as follows. ${ }^{4}$ Endow each $X \in Y$ with the discrete topology. Since $X$ is finite it is a compact $T_{2}$-space in this topology. Hence $\prod_{x \in Y} X$ is a compact $T_{2}$-space in the prod-

3 The failure of this implication in the context of Zermelo-Fraenkel set theory with weakened forms of regularity was demonstrated in [4].

${ }^{4}$ The Tychonoff theorem for $T_{2}$-spaces is equivalent to the Boolean prime ideal theorem in the Zermelo-Fraenkel context (see [6]). Also, Philip Hall's theorem for finite families is provable in this context.

The implication here stated was known to Dana Scott and is undoubtedly known or obvious to logicians who work with the compactness theorem. For the orientation which makes such theorems obvious one may refer either to Leon Henkin [5] or to J. Łos and C. Ryll-Nardzewski [7]. 
uct topology. Let $F$ be the family of all sets

$$
S_{G}=\left\{f \in \prod_{X \in Y} X: f \uparrow G \text { is } 1-1\right\}
$$

where $G$ ranges over finite subsets of $Y . S_{G}$ is closed, and nonempty by Philip Hall's theorem for finite families, and for any $G_{1}, G_{2}, S_{G_{1}} \cap S_{G_{2}}$ $\supseteq S_{G_{1} \cup G_{2}}$. Hence by compactness $\cap F$ is nonempty. But any $f \in \cap F$ is a $1-1$ choice function. ${ }^{5}$ Q.E.D.

\section{REFERENCES}

1. M. N. Bleicher, Some theorems on vector spaces and the axiom of choice, Fund. Math. 54 (1964), 95-197.

2. M. Hall, Combinatorial mathematics, Blaisdell, New York, 1966.

3. J. D. Halpern, Bases in vector spaces and the axiom of choice, Abstract 622-672, Notices Amer. Math. Soc. 12 (1965), 348.

4. - The independence of the axiom of choice from the Boolean prime ideal theorem, Fund. Math. 55 (1964), 57-66.

5. L. Henkin, Some interconnections between modern algebra and mathematical logic, Trans. Amer. Math. Soc. 74 (1953), 410-427.

6. J. Los and C. Ryll-Nardzewski, Effectiveness of the representation theorem for Boolean algebras, Fund. Math. 41 (1954-55), 49-56.

7. - On the application of Tychonoff's theorem in mathematical proofs, Fund. Math. 38 (1951), 233-237.

8. O. Zariski and P. Samuel, Commutative algebra, Vol. 1, Van Nostrand, Princeton, New Jersey, 1958.

\section{California Institute of Technology}

5 A direct proof from Philip Hall's theorem and the Boolean prime ideal theorem (actually, the dual ultrafilter theorem) is as follows. Let $S$ be the set of all 1-1 functions with domain included in $Y$ and such that $f(X) \in X$ all $X \in D f$. Let $B$ be the set algebra of all subsets of $S$ and let $I$ be the filter generated by the sets, $F_{X}=\{f: X \in D f\}$, all $X \in Y . I$ is proper by Philip Hall's theorem. Let $J$ be an ultrafilter including $I$. Then for each $X \in Y$ there is a unique $x \in X$ such that $\{f: f(X)=x\} \in J$. This correspondence defines the required function. 